\title{
ОПТИМИЗАЦИЯ ПЕРЕНОСА ОДНОГО ЭМБРИОНА У ПАЦИЕНТОК С ХОРОШИМ ОВАРИАЛЬНЫМ РЕЗЕРВОМ
}

Н. В. Сараева ${ }^{1,2}$, Н. В. Спиридонова ${ }^{1}$, М. Т. Тугушев ${ }^{1}$, О. В. Шурыгина ${ }^{1}$, А. И. Синицына ${ }^{1}$ А. О. Корчагин²

${ }^{1}$ Самарский государственный медицинский университет, Самара, Россия

2 «Медицинская компания ИДК», группа компаний «Мать и Дитя», Самара, Россия

Совершенствование вспомогательных репродуктивных технологий привело к росту числа случаев многоплодной беременности. Один из инструментов выбора качественного эмбриона на перенос - использование time-lapse микроскопии (TLM). Целью работы было оценить исходы переноса одного эмбриона на пятые сутки культивирования у пациенток с хорошим овариальным резервом в программе ЭКО с использованием TLM. Исследовали 208 женщин с бесплодием, с хорошим овариальным резервом (при пункции фолликулов получено более восьми ооцитов): у 95 пациенток провели перенос одного эмбриона с использованием системы TLM (группа исследования); у 113 пациенток - с использованием традиционного культивирования и выбора эмбриона для переноса (группа контроля). Проведена оценка качества переносимых эмбрионов, частоты наступления клинической беременности, частоты достижения родов и случаев потери беременности. В каждой группе выделены две подгруппы: с неэлективным переносом одного эмбриона (подгруппа 5SET: 45 пациенток в группе исследования, 67 - в контрольной) и с элективным (подгруппа 5 eSET: 50 пациенток в группе исследования, 46 - в контрольной). Группы не различались по среднему возрасту, фактору бесплодия, длительности бесплодия. В группе исследования в $100 \%$ случаев перенесены эмбрионы хорошего и отличного качества, в группе контроля - в 93,8\% ( $p=0,037)$. Частота наступления клинической беременности составила 64,2\% в основной группе и 60,2\% - в контрольной ( $p=0,65)$. В группе исследования частота родов составила 54\% в подгруппе 5еSET и $51,1 \%$ - в подгруппе 5SET ( $p=0,940)$. В группе контроля в подгруппе 5eSET частота родов составила 54,4\%, а в подгруппе 5SET — 34,3\% ( $p=0,052$ по методу Фишера). Проведение элективного переноса эмбриона (5eSET) или использование TLM повышало вероятность родов в 2,17 раза ( $p$ = 0,01).

Ключевые слова: вспомогательные репродуктивные технологии, перенос одного эмбриона, элективный перенос бластоцисты, time-Іарsе микроскопия Благодарности: к.б.н., доценту Самарского национального исследовательского университета имени академика С. П. Королева М. В. Комаровой за помощь в статистической обработке результатов исследования.

Вклад авторов: все авторы внесли равнозначный вклад в исследование и написание статьи.

Соблюдение этических стандартов: исследование одобрено этическим комитетом СамГМУ (протокол № 194 от 12 сентября 2018 г.). Всеми пациентами подписано добровольное информированное согласие на лечение методами вспомогательных репродуктивных технологий.

$\triangle$ Для корреспонденции: Наталья Владимировна Сараева

ул. Врубеля, 15-131, г. Самара, 443086; kuzichkina@gmail.com

Статья получена: 19.03.2020 Статья принята к печати: 07.04.2020 Опубликована онлайн: 20.04.2020

DOI: $10.24075 /$ vrgmu.2020.021

\section{OPTIMIZATION OF A SINGLE-EMBRYO TRANSFER IN PATIENTS WITH GOOD OVARIAN RESERVE}

\author{
Saraeva NV ${ }^{1,2}$, Spiridonova NV ${ }^{1}$, Tugushev MT11 , Shurygina OV ${ }^{1}$, Sinitsyna $\mathrm{Al}^{1}$, Korchagin $\mathrm{AO}^{2}$ \\ ${ }^{1}$ Samara State Medical University, Samara, Russia \\ ${ }^{2}$ IDK Medical Company, the Mother and Child group, Samara, Russia
}

Due to refinements of assisted reproductive technology, the number of multiple pregnancies has increased substantially. Time-lapse microscopy (TLM) is a tool for selecting quality embryos for transfer. This study aimed to assess the outcomes of single-embryo transfer of autologous oocytes performed on day 5 of embryo incubation in a TLM-equipped system in patients with good ovarian reserve. The study was carried out in 208 infertile women with good ovarian reserve (over 8 oocytes retrieved). Single-embryo transfer following incubation in a TLM-equipped incubator was performed in 95 patients, who formed the main group; the control group consisted of 113 patients undergoing single-embryo transfer following a traditional culture and embryo selection procedure. We assessed the quality of transferred embryos, the rates of clinical pregnancy and pregnancy loss. Two subgroups were identified in each group of the participants: the 5SET subgroup (nonelective single-embryo transfer), which included 45 patients from the main group and 67 controls, and the 5 eSET subgroup (elective single-embryo transfer), which consisted of 50 main group patients and 46 controls. The groups did not differ in terms of age, infertility factors and infertility duration. The quality of transferred embryos was excellent or good in all main group patients (100\%); in the control group, the quality of transferred embryos was excellent or good in $93.8 \%$ of cases $(p=0.037)$. Clinical pregnancies were achieved in $64.2 \%$ of women in the main group and in $60.2 \%$ of controls $(p=0.65)$. Delivery rates were $54 \%$ and $51.1 \%$ in the 5 eSET and 5SET subgroups of the main group, respectively $(p=0.940)$. For the control group, delivery rates were $54.4 \%$ and $34.3 \%$ in the 5 eSET and 5SET subgroups, respectively $(p=0.052$, Fisher exact test). Elective single-embryo transfer (5eSET) and the use of TLM increased the chance of pregnancy 2.17 -fold $(p=0.01)$.

Keywords: assisted reproductive technology, single-embryo transfer, elective blastocyst transfer, time-lapse microscopy

Acknowledgements: the authors thank Komarova MV, PhD Biol., Associate Professor of Samara National Research University, for her help with the statistical analysis. Author contribution: all authors equally contributed to the study and manuscript preparation.

Compliance with ethical standards: the study was approved by the Ethics Committee of Samara State Medical University (Protocol 194 dated September 12 , 2018). Informed consent was obtained from all study participants.

$\triangle$ Correspondence should be addressed: Natalya V. Saraeva Vrubelya, 15-131, Samara, 443086; kuzichkina@gmail.com

Received: 19.03.2020 Accepted: 07.04.2020 Published online: 20.04.2020

DOI: 10.24075/brsmu.2020.021 
Совершенствование вспомогательных репродуктивных технологий (ВРТ) за последние 15-20 лет привело к повышению частоты имплантации эмбрионов человека, что повлекло за собой рост числа многоплодных беременностей. Многоплодная беременность является доказанным фактором риска осложненного течения беременности и родов и значительно повышает риск возникновения материнских и перинатальных осложнений [1-3]. В связи с этим перенос одного эмбриона стал приоритетной задачей лечения методами ВРТ [4, 5].

Для максимального повышения вероятности наступления беременности в программах ВРТ первостепенное значение имеет возможность выбора эмбриона с наивысшим потенциалом развития. Это позволит сократить время до достижения беременности и облегчит ранжирование оставшихся эмбрионов для криоконсервации, что в последующем будет способствовать переносу потенциально качественного эмбриона в первую очередь [6, 7].

C момента возникновения технологии экстракорпорального оплодотворения (ЭКО) морфологическая оценка эмбрионов человека была основным методом, используемым эмбриологами для оценки развития и выбора эмбрионов при переносе. Со временем были разработаны классификации для оценки развития и жизнеспособности эмбрионов. Однако их использование на практике оказалось более трудным, чем ожидалось, из-за высокодинамичного характера развития эмбриона в период преимплантации. Другими словами, эмбрион, оцененный в 8 ч утра на вторые сутки развития, может выглядеть совсем по-другому через несколько часов [8]. Следовательно, чрезвычайно сложно интерпретировать данные морфологии без оценки развития эмбриона в динамике, с анализом морфологии в разные промежутки времени.

С внедрением в лабораторию ЭКО time-lapse технологии, или TLM (электронной микроскопии с временным интервалом), - современного метода выбора эмбриона для переноса, наступила новая эра эмбриологии. Благодаря этой методике на сегодняшний день возможна оценка морфологии эмбрионов как континуум, т. е. с фиксацией изображения каждые несколько минут [9-11].

В настоящее время получены противоречивые данные о результатах использования TLM. Ретроспективное исследование показало, что культивирование эмбрионов в инкубаторе EmbryoScope может улучшить показатели рождаемости, а традиционные методы культивирования негативно влияют на развитие эмбрионов и потенциал имплантации [12]. Авторы текущих ретроспективных и проспективных исследований подчеркивают и преимущество этой технологии с многообещающими результатами [13-16], и отсутствие различий по сравнению с морфологической оценкой качества эмбрионов $[17,18]$.

Целью исследования было оценить исходы переноса одного эмбриона в программе ЭКО у пациенток с хорошим овариальным резервом с использованием TLM.

\section{ПАЦИЕНТЫ И МЕТОДЫ}

В исследование были включены 208 женщин с бесплодием, которым был проведен перенос одного эмбриона по программе ЭКО на базе ЗАО «Медицинская компания ИДК» (г. Самара) в 2013-2015 гг.

Произведен статистический анализ 208 клинических и эмбриологических протоколов пациенток с помощью статистического пакета SPSS21, номер лицензии
20130626-3 (An IBM Company; CШA) и Microsoft Excel (Microsoft; CША).

Критерии включения в исследование: программа ЭКО; циклы с использованием собственных ооцитов; циклы с получением восьми и более ооцитов; перенос одного эмбриона на пятые сутки культивирования; толщина эндометрия 8 мм и более на день переноса эмбриона.

Критерии исключения: программа ИКСИ; циклы с использованием донорских ооцитов; циклы с получением менее 8 ооцитов; циклы с переносом размороженного эмбриона; перенос эмбрионов на третьи сутки культивирования; перенос двух эмбрионов; толщина эндометрия менее 8 мм на день переноса эмбриона.

Ограничений по возрасту пациенток для включения в исследование не было. Минимальный возраст составил 20 лет, максимальный - 42 года.

Пациенток разделили на две группы. Группу исследования составили 95 пациенток с хорошим овариальным резервом с переносом одного эмбриона по программе ЭКО с использованием системы time-lapse микроскопии.

Группу контроля составили 113 пациенток с хорошим овариальным резервом с переносом одного эмбриона по программе ЭКО с использованием метода традиционного культивирования и выбора эмбриона для переноса. Средний возраст, факторы бесплодия, длительность отсутствия бесплодия в браке, порядковый номер настоящей программы ЭКО статистически не различались у пациенток двух групп. Средний возраст женщин в основной и контрольной группах составил 31,40 \pm 0,38 и 30,65 \pm 0,37 г. соответственно $(p>0,05)$.

Эмбриологический этап программы ЭКО в группе исследования проводили с использованием видеосистемы наблюдения за развитием эмбрионов Primovision (Vitrolife; Швеция).

В обеих группах для оценки качества эмбрионов использовали буквенно-цифровую систему, разработанную Gardner и Schoolcraft в 1999 г. [19]. Бластоцисты градации AA, AB, ВА оценивали как эмбрионы отличного качества; бластоцисты градации ВВ оценивали как эмбрионы хорошего качества; бластоцисты градации АС, СА, ВС, СВ, СС оценивали как эмбрионы удовлетворительного качества.

По типу переноса эмбриона в каждой группе были выделены две подгруппы: подгруппа с неэлективным переносом одного эмбриона на пятые сутки культивирования (подгруппа 5SET: 45 пациенток в группе исследования, 67 - в группе контроля) и подгруппа с элективным переносом одного эмбриона на пятые сутки культивирования (подгруппа 5eSET: 50 пациенток в группе исследования, 46 - в группе контроля). Элективным считали перенос при наличии выбора из двух и более эмбрионов отличного качества.

Дополнительно в группе исследования выбор эмбрионов на перенос проводили на основании их соответствия ключевым морфодинамическим параметрам системы. В качестве ключевых событий деления эмбрионов оценивали следующие параметры: время наступления первого дробления; интервал времени между первым и вторым дроблением; время наступления второго дробления; время наступления третьего дробления; время формирования бластоцисты. Если все параметры деления эмбриона соответствовали референсным значениям системы и эмбрион был отличного или хорошего качества на основании морфологической оценки, такой эмбрион выбирали для переноса (референс-положительный 


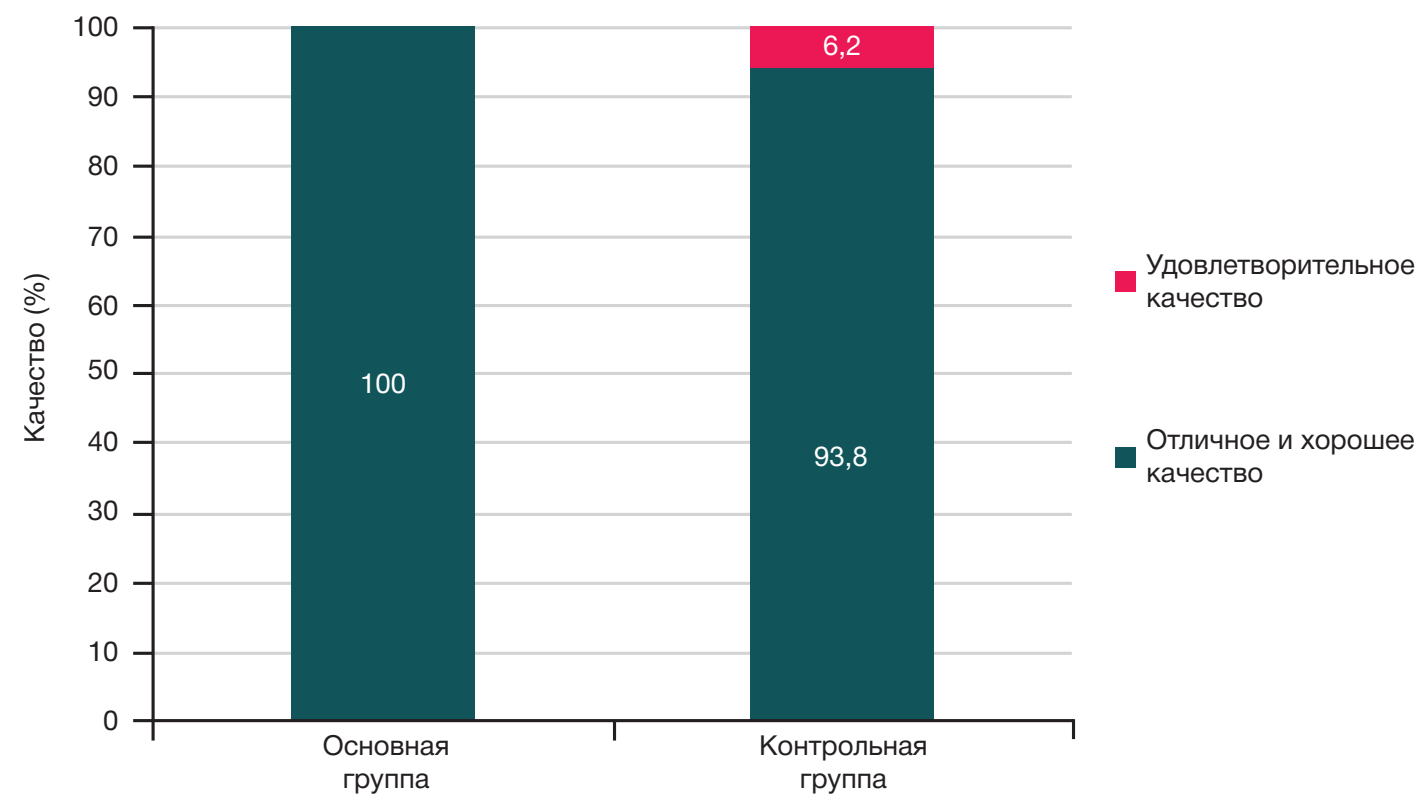

Рис. 1. Качество эмбрионов у пациенток основной группы и группы контроля

эмбрион). В подгруппе с референс-положительным эмбрионом было 52 пациентки. В том случае, если один или несколько параметров деления эмбриона не соответствовало референсному значению системы, выбор эмбриона на перенос осуществляли дополнительно на основании стандартной морфологической оценки (референс-отрицательный эмбрион). В подгруппе с референс-отрицательным эмбрионом было 43 пациентки.

В обеих группах культивирование проводили с использованием универсальной среды Continuous Single Culture (Irvine Scientific; USA). Оценку качества эмбрионов на пятые сутки культивирования проводили через 116-118 ч после оплодотворения.

\section{РЕЗУЛЬТАТЫ ИССЛЕДОВАНИЯ}

В ходе исследования в обеих группах была проведена оценка качества переносимых эмбрионов, частоты наступления клинической беременности, частоты наступления родов и частоты случаев потери беременности.

Доля пациенток позднего репродуктивного возраста ( $\geq 35$ лет) составила 21,05\% в основной группе и 23,89\% в группе контроля (р > 0,05). Поскольку настоящее исследование включало только женщин с хорошим овариальным резервом и с переносом одного эмбриона, то основную часть наблюдений составили женщинь молодого репродуктивного возраста.
Среднее число полученных ооцитов в основной и контрольной группах составило 11,87 \pm 0,32 и 12,49 \pm 0,40 соответственно ( $p>0,05)$.

Известно, что качество переносимых эмбрионов значительно влияет на вероятность наступления беременности при использовании ЭКО. Так, при переносе эмбрионов отличного или хорошего качества показатель частоты наступления клинической беременности значительно выше, чем при переносе эмбрионов удовлетворительного качества [20]. В группе исследования в 100\% случаев были перенесены эмбрионы хорошего (16 эмбрионов) и отличного (79 эмбрионов) качества, в группе контроля этот показатель составил 93,8\% (из них 88 эмбрионов отличного качества, 18 эмбрионов хорошего качества) ( $p=0,037$ ) (рис. 1). У 7 пациенток группы контроля были перенесены эмбрионы удовлетворительного качества (6,2\%).

При анализе качества переносимых эмбрионов между подгруппой пациенток с положительным референсным значением перенесенных эмбрионов и подгруппой пациенток с отрицательным референсным значением перенесенных эмбрионов различий не выявлено. В подгруппе с положительным референсным значением доля циклов с переносом отличного эмбриона составила $87,5 \%$, в подгруппе с отрицательным референсным значением - 78,95\% $(p=0,44)$.

Таким образом, частота наступления клинической беременности при использовании ЭКО не различалась

Таблица 1. Исходы переноса эмбрионов у пациенток основной группы и группы контроля

\begin{tabular}{|c|c|c|c|c|c|c|c|}
\hline & & \multicolumn{2}{|c|}{ Основная группа } & \multicolumn{2}{|c|}{ Контрольная группа } & \multirow[t]{2}{*}{$\chi^{2}$} & \multirow[t]{2}{*}{$p$} \\
\hline & & Абс. & $\%$ & Абс. & $\%$ & & \\
\hline \multirow{2}{*}{ Беременность } & Нет & 34 & $35,8 \%$ & 45 & $39,8 \%$ & \multirow[t]{2}{*}{0,2} & \multirow[t]{2}{*}{0,65} \\
\hline & Да & 61 & $64,2 \%$ & 68 & $60,2 \%$ & & \\
\hline \multirow{5}{*}{ Исходы } & Нет беременности & 34 & $35,8 \%$ & 45 & $39,8 \%$ & \multirow[t]{5}{*}{3,7} & \multirow[t]{5}{*}{0,443} \\
\hline & Ранние потери & 11 & $11,6 \%$ & 18 & $15,9 \%$ & & \\
\hline & Поздние потери & & & 2 & $1,8 \%$ & & \\
\hline & Преждевременные роды & 1 & $1,1 \%$ & 1 & $0,9 \%$ & & \\
\hline & Срочные роды & 49 & $51,6 \%$ & 47 & $41,6 \%$ & & \\
\hline \multirow{2}{*}{ Роды } & Нет & 45 & $47,4 \%$ & 65 & $57,5 \%$ & \multirow[t]{2}{*}{1,7} & \multirow[t]{2}{*}{0,186} \\
\hline & Да & 50 & $52,6 \%$ & 48 & $42,5 \%$ & & \\
\hline
\end{tabular}




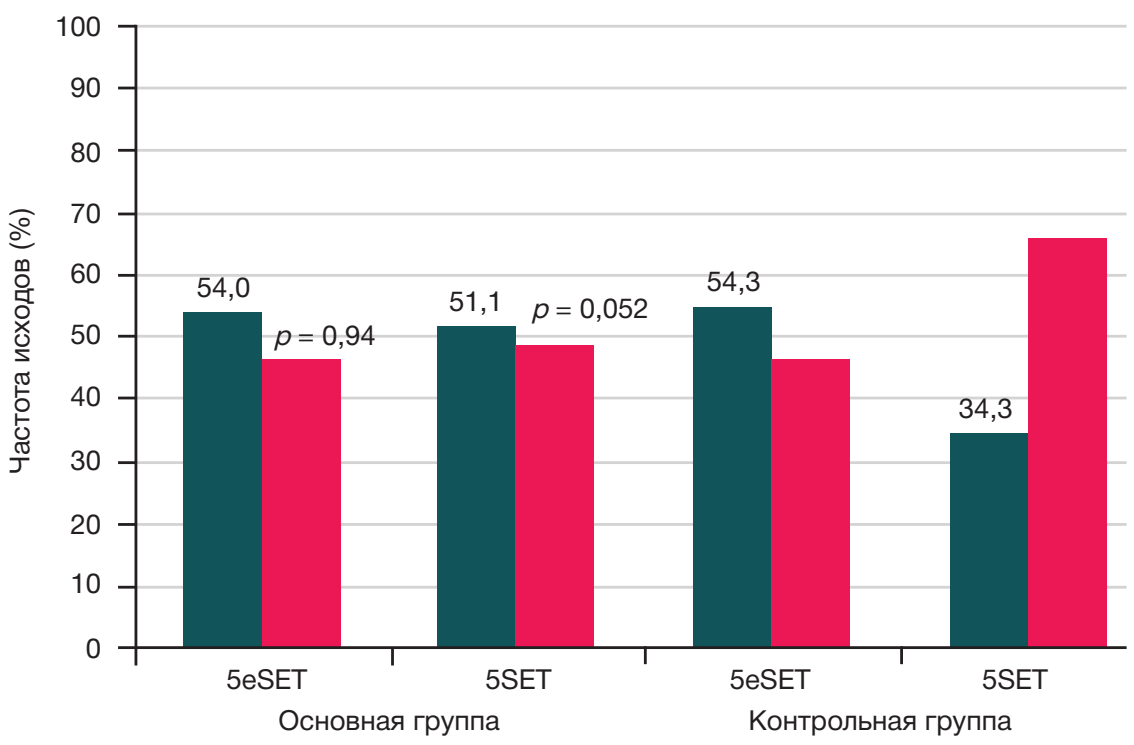

Роды есть

Родов нет

Рис. 2. Частота родов в зависимости от вида переноса эмбриона

между двумя группами и составила 64,2\% в основной группе и 60,2\% в группе контроля ( $p=0,65)$ (табл. 1).

Среди всех исходов переноса эмбрионов доля наступивших родов составила $52,6 \%$ в основной группе и 42,5\% в группе контроля ( $>$ > 0,05). Число всех случаев ранней потери беременности (биохимические беременности и потери в сроке до 12 недель гестации) в основной группе составило 11,6\%, в группе контроля $17,7 \%$, хотя разница была статистически незначимой.

Мы не получили статистически значимых различий в группе исследования между подгруппой пациенток с положительным референсным значением перенесенных эмбрионов и подгруппой пациенток с отрицательным референсным значением перенесенных эмбрионов в частоте клинической беременности (66,7 и 60,5\% соответственно) и по частоте наступивших родов (50 и 52,6\% соответственно).

Так как по данным литературы элективный перенос эмбриона повышает вероятность благоприятного исхода переноса [21], был проведен сравнительный анализ частоты наступивших родов в зависимости от вида переноса.

Так, в основной группе частота родов составила 54\% при элективном переносе эмбриона (подгруппа 5eSET) и 51,1\% - при неэлективном переносе (подгруппа 5SET) ( существенно влиял на частоту родов: в подгруппе переноса 5eSET частота родов составила 54,3\%, а в подгруппе переноса 5SET — только 34,3\% ( $p=0$ 0,052 при оценке точным методом Фишера). Разница в частоте родов составила 20,1\% (95\% ДИ - 1,5-37\%) при ОШ 2,28 (95\% ДИ 1,06-4,91). Таким образом, у женщин при использовании TLM независимо от вида переноса процент наступления родов был высоким 54,0\% и 51,1\% и статистически не различался.

С учетом полученных выше данных нами была проведена оценка связи благоприятного исхода цикла
ЭКО (наступление родов) с двумя факторами: наличием или отсутствием TLM и с видом переноса эмбриона (элективный или неэлективный) (табл. 2).

В группе комбинации двух признаков (вид переноса 5eSET в обеих группах и вид переноса 5SET в основной группе) частота наступления родов достигала 53,2\%, в то время как в группе контроля с видом переноса 5SET этот показатель был ниже и составил 34,3\% ( $p=0,01)$ (ОШ = 2,17 (1,19-3,97)). Таким образом, можно предположить, что есть положительная тенденция к повышению частоть наступления родов при использовании TLM независимо от вида переноса эмбриона.

\section{ОБСУЖДЕНИЕ РЕЗУЛЬТАТОВ}

Технология использования TLM минимизирует контакт эмбриона с внешней средой, что может быть одним из факторов, обусловливающих более высокий имплантационный потенциал эмбриона. Непрерывный мониторинг с короткими промежутками времени дает больше информации о кинетике и морфологии эмбрионов по сравнению со стандартной ежедневной оценкой. Текущие публикации содержат противоречивые данные о влиянии TLM на результаты программы ЭКО.

В рамках нашего исследования получены данные o переносе бластоцист более высокого качества в группе TLM, что совпадает с данными ряда авторов $[22,23]$. Так, у пациенток с хорошим овариальным резервом доля бластоцист хорошего качества и число криоконсервированных эмбрионов на пациентку были существенно ниже в группе контроля, по сравнению с

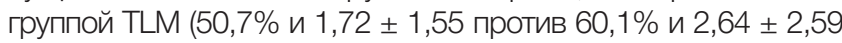
соответственно; $p<0,05) ;$ хотя не было получено статистически значимых различий в числе эмбрионов хорошего качества на третьи сутки, частоте случаев наступления клинической беременности и частоте случаев

Таблица 2. Частота наступления родов в зависимости от использования TLM и вида переноса эмбриона

\begin{tabular}{|c|c|c|c|c|c|c|}
\hline \multirow{3}{*}{ Роды } & \multicolumn{4}{|c|}{ Вид переноса } & \multirow{3}{*}{$x^{2}$} & \multirow{3}{*}{$p$} \\
\hline & \multicolumn{2}{|c|}{ 5eSET в обеих группах + 5SET в основной группе } & \multicolumn{2}{|c|}{ 5SET в группе контроля } & & \\
\hline & Абс. & $\%$ & Абс. & $\%$ & & \\
\hline Нет & 66 & $46,8 \%$ & 44 & $65,7 \%$ & 5,75 & 0,01 \\
\hline Да & 75 & $53,2 \%$ & 23 & $34,3 \%$ & & \\
\hline
\end{tabular}


имплантации [23]. Поскольку в нашем исследовании пациентки по клиническим характеристикам (возрасту, факторам бесплодия, продолжительности бесплодия) не различались между двумя группами, можно предположить, что различие в доле эмбрионов отличного и хорошего качества связано с отсутствием влияния в группе TLM факторов внешней среды (температуры, света, изменения рН).

Эффективность TLM может быть обусловлена двумя факторами: стабильными условиями культивирования эмбрионов (для оценки качества эмбриона нет необходимости извлекать эмбрион из инкубатора) и возможностью выбора эмбриона на перенос с помощью программного обеспечения [24].

По результатам недавно проведенного обзора Cochrane (2995 супружеских пар), отсутствуют убедительные доказательства преимущества TLM по сравнению с традиционным культивированием - не было обнаружено достоверных различий в частоте случаев наступления клинической беременности (ОШ 0,95; 95\% ДИ - 0,781,16) и частоте родов (ОШ 1,12; 95\% ДИ - 0,92-1,36) [25]. В метаанализе ведения 1637 пациенток, напротив, показано преимущество использования TLM по сравнению со стандартной инкубацией и оценкой качества эмбрионов [26]. В этом исследовании сообщается о высокой частоте наступления клинической беременности (51,0 против 39,9\%; ОШ 1,54, 95\% ДИ - 1,21-1,97), более низкой частоте ранней потери беременности (15,3 против 21,3\%; ОШ 0,66, 95\% ДИ - 0,47-0,94) и высокой частоте случаев живорождения (44,2 против 31,3\%; ОШ 1,67, 95\% ДИ $1,13-2,46)$.

Мы получили высокую частоту наступления клинической беременности в обеих группах $(64,2 \%$ в группе исследования и 60,2\% в контрольной группе), что может свидетельствовать об отсутствии негативного влияния на культуру эмбрионов использования системы непрерывного мониторинга. Применение покадровой микроскопии вело к снижению числа случаев ранней потери беременности.

Нельзя исключить, что отсутствие различий между двумя группами по частоте наступления клинической беременности, частоте наступления родов и случаев ранней потери беременности в нашем исследовании может быть связано с небольшим с точки зрения статистики числом наблюдений (95 пациенток) с помощью TLM.

В одном из ретроспективных когортных исследований было показано увеличение частоты случаев наступления клинической беременности в группе TLM (+15,7\% на перенос эмбриона) [27]. Но в отличие от нашей работы, это исследование было гетерогенным по составу пациентов (включало циклы с донорскими ооцитами), количеству переносимых эмбрионов (от 1 до 3 эмбрионов), по суткам переноса - в большинстве случаев в группе TLM проводили перенос на третьи сутки культивирования, что снижало итоговый показатель частоты наступления клинической беременности. Так, в указанной работе в группе TLM показатель наступления клинической беременности на пункцию фолликулов (получение ооцитов) при переносе эмбриона на пятые сутки культивирования составил 50\%, в то время как в нашем исследовании показатель наступления клинической беременности в расчете на перенос эмбриона на пятые сутки культивирования достигал $64,2 \%$. Из достоинств этой работы можно отметить разработанную авторами прогностическую (математическую) модель, согласно которой в клинике ВРТ после использования TLM можно ожидать увеличения числа случаев наступления клинической беременности на 15,7\% в расчете на перенос эмбриона. При этом улучшения результатов лечения чаще всего можно достигнуть увеличением числа циклов ЭКО с использованием TLM до 200 раз и выше.

Как многие технологические достижения, применение TLM может не дать быстрого эффекта во всех лабораториях, и для успешного внедрения, вероятно, потребуется некоторая стандартизация. Действительно, TLM не всегда имеет преимущество при отборе эмбрионов в клинических исследованиях [22]. Однако нельзя не упомянуть о ее возрастающей ценности для проведения непрерывного культивирования и помощи в выборе времени процедуры биопсии эмбриона [28, 29].

В настоящее время ведутся работы по внедрению искусственного интеллекта в TLM для определения комбинаций параметров, способных указать на максимальный потенциал эмбриона к имплантации и живорождению [24].

\section{ВЫВОДЬ}

В проведенном исследовании не выявлено различий в частоте наступления клинической беременности, частоте достижения родов и частоте случаев ранней потери беременности между группой TLM и группой традиционного культивирования эмбрионов, что может быть связано с небольшим числом наблюдений в группе TLM. При использовании TLM выявлена высокая частота наступления родов вне зависимости от элективного и неэлективного переноса эмбриона, однако отсутствовали различия в частоте случаев наступления клинической беременности и ранней потери беременности. При использовании традиционного способа культивирования и выбора эмбриона для переноса вид переноса существенно влиял на частоту случаев достижения родов: в подгруппе элективного переноса показатель родов был выше, по сравнению с подгруппой неэлективного переноса ( $p=0,052$ при оценке точным методом Фишера). Проведение элективного переноса эмбриона на пятые сутки культивирования (5eSET) или использование TLM вне зависимости от вида переноса было благоприятным фактором и повышало шанс на роды $(p=0,01)$.

Представленные данные открывают перспективы изучения преимущества использования TLM у пациенток разных возрастных групп, у пациенток со сниженным овариальным резервом. Дальнейшее накопление фактического материала необходимо для анализа таких показателей, как кумулятивный показатель беременности в циклах ВРТ с использованием системы видеонаблюдения, а также для мониторинга отдаленных результатов использования этой технологии. Нет сомнений, что скоро будут созданы сложные системы для неинвазивной оценки качества эмбрионов (морфологии, динамики и метаболизма), позволяющие автоматизировать процесс выбора эмбриона для переноса. Они снизят вероятность воздействия фракторов человека и окружающей среды и, как следствие, увеличат рождаемость при переносе эмбрионов. 
1. Калинкина О. Б., Спиридонова Н. В., Юнусова Ю. Р. Аравина О. Р. Многофакторный анализ риска развития акушерских и перинатальных осложнений у пациенток с ожирением и избыточной массой тела. Известия Самарского научного центра РАН. 2015; 17 (5-3): 793-7.

2. Anderson P, Doyle LW. Victorian Infant Collaborative Study Group. Neurobehavioral outcomes of school-age children born extremely low birth weight or very preterm in the 1990s. J Am Med Assoc. 2003; 289: 3264-72.

3. Ombelet W, De Sutter P, Van der Elst J, Martens G. Multiple gestation and infertility treatment: registration, reflection and reaction-the Belgian project. Hum Reprod Update. 2005; 11: 3-14.

4. De Sutter P, Van der Elst J, Coetsier T, Dhont M. Single embryo transfer and multiple pregnancy rate reduction in IVF/ICSI: a 5-year appraisal. Reprod Biomed Online. 2003; 7: 464-9.

5. Thurin A, Hausken J, Hillensjo T, Jablonowska B, Pinborg A Strandell A, Bergh C. Elective single-embryo transfer versus double-embryo transfer in in vitro fertilization. N Engl J Med. 2004; 351: 2392-402.

6. Bergh C. Single embryo transfer: a mini-review. Hum Reprod. 2005; 20: 323-7.

7. Gardner DK, Meseguer M, Rubio C, Treff NR. Diagnosis of human preimplantation embryo viability. Hum Reprod Update. 2015; 21: 727-47.

8. Bavister BD. Culture of preimplantation embryos: facts and artifacts. Hum Reprod Update. 1995; 1: 91-148.

9. Meseguer M. Time-lapse: the remaining questions to be answered. Fertil Steril. 2016; 105 (2): 295-6.

10. Pribenszkya C, Matyasb S, Kovacsb P, Losonczic E, Zádori J Vajta G. Pregnancy achieved by transfer of a single blastocyst selected by time-lapse monitoring. Reprod Biomed Online. 2010; 21 (4): 533-6.

11. Wang SX. The past, present, and future of embryo selection in in vitro fertilization: Frontiers in Reproduction Conference. Yale J Biol Med. 2011; 84 (4): 487-90.

12. McEvoy K, Brison D, Roberts S, Turner C, Adeniyi T, Wood L, et al. A one year retrospective analysis comparing live birth outcomes from embryos grown and transferred from an undisturbed timelapse culture system with a conventional culture system. Human reproduction. 2016; 31: i174-i175.

13. Шурыгина О. В., Сараева Н. В., Тугушев М. Т., Пекарев В. А. Байзарова А. А., Краснова О. В., и др. Отдаленные результать программ ВРТ с использованием системы непрерывного видеонаблюдения на эмбриологическом этапе. В сборнике: Репродуктивные технологии сегодня и завтра. Материаль XXVII международной конференции Российской ассоциации репродукции человека; 6-9 сентября 2017 г.; М.: Медиа Cфера, 2017; 328 c.

14. Adamson GD, Abusief ME, Palao LM, Gvakharia M. Improved implantation rates of day 3 embryo transfers with the use of an automated time-lapse-enabled test to aid in embryo selection. Fertil Steril. 2015; 105: 369-75.

15. Diamond MP, Suraj V, Behnke EJ, Yang X, Angle MJ, LambeSteinmiller JC, et al. Using the Eeva Test ${ }^{\mathrm{TM}}$ adjunctively to traditional day 3 morphology is informative for consistent embryo assessment within a panel of embryologists with diverse

\section{References}

1. Kalinkina OB, Spiridonova NV, Junusova JuR, Aravina OR Mnogofaktornyj analiz riska razvitija akusherskih i perinatal'nyh oslozhnenij u pacientok s ozhireniem i izbytochnoj massoj tela. Izvestija Samarskogo nauchnogo centra RAN. 2015; 17 (5-3): 793-7.

2. Anderson P, Doyle LW. Victorian Infant Collaborative Study Group. Neurobehavioral outcomes of school-age children born extremely low birth weight or very preterm in the 1990s. J Am Med Assoc. 2003; 289: 3264-72. experience. J Assist Reprod Genet. 2015; 32: 61-68.

16. Ver Milyea MD, Tan Lei, Joshua TA, Conaghan J, Ivani $K$, Gvakharia $\mathrm{M}$, et al. Computer-automated time-lapse analysis results correlate with embryo implantation and clinical pregnancy: A blinded, multi-centre study. Reprod Biomed Online. 2014; 29 (6): 729-36.

17. Kirkegaard K, Hindkjaer JJ, Grøndahl ML, Kesmodel US, Ingerslev HJ. A randomized clinical trial comparing embryo culture in a conventional incubator with a time-lapse incubator. J Assist Reprod Genet. 2012; 29 (6): 565-72.

18. Minasi MG, Colasante A, Riccio T, Ruberti A, Casciani V, Scarselli F, et al. Correlation between aneuploidy, standard morphology evaluation and morphokinetic development in 1730 biopsied blastocysts: a consecutive case series study. Hum Reprod. 2016; 31 (10): 2245-54.

19. Gardner DK, Schoolcraft WB. Culture and transfer of human blastocysts. Curr Opin Obstet Gynecol. 1999 Jun; 11 (3): 307-11.

20. Gardner DK, Lane M, Stevens J, Schlenker T, Schoolcraft WB. Blastocyst score affects implantation and pregnancy outcome: towards a single blastocyst transfer. Fertil Steril. 2000; 73: 1155-8.

21. Lee AM, Connell MT, Csokmay JM, Styer AK. Elective single embryo transfer - the power of one. Contracept Reprod Med. 2016; 1: 11.

22. Goodman LR, Goldberg J, Falcone T, Austin C, Desai N. Does the addition of time-lapse morphokinetics in the selection of embryos for transfer improve pregnancy rates? A randomized controlled trial. Fertil Steril. 2016; 105 (2): 275-85.

23. Zhang JQ, Li XL, Peng Y, Guo X, Heng BC, Tong GQ. Reduction in exposure of human embryos outside the incubator enhances embryo quality and blastulation rate. Reprod Biomed Online. 2010; 20: 510-15.

24. Apter S, Ebner T, Freour T, Guns Y, Kovacic B, Le Clef N, et al. Good practice recommendations for the use of time-lapse technology. Human Reproduction Open. 2020; 2020 (2: hoaa008. Available from: https://doi.org/10.1093/hropen/hoaa008.

25. Armstrong S, Bhide P, Jordan V, Pacey A, Marjoribanks J, Farquhar C. Time-lapse systems for embryo incubation and assessment in assisted reproduction. Cochrane Database of Systematic Reviews. 2018; 25 (5): CD011320. DOI: 10.1002/14651858. CD011320.pub4.

26. Pribenszkya C, Nilselid AM, Montag M. Time-lapse culture with morphokinetic embryo selection improves pregnancy and live birth chances and reduces early pregnancy loss: a meta-analysis. Reprod Biomed Online. 2017 Nov; 35 (5): 511-520.

27. Meseguer M, Rubio I, Cruz M, Basile N, Marcos J, Requena A. Embryo incubation and selection in a time-lapse monitoring system improves pregnancy outcome compared with a standard incubator: a retrospective cohort study. Fertil Steril. 2012 Dec; 98 (6): 1481-9.

28. Athayde Wirka K, Chen AA, Conaghan J, Ivani K, Gvakharia M, Behr B, et al. Atypical embryo phenotypes identified by timelapse microscopy: high prevalence and association with embryo development. Fertil Steril. 2014; 101 (6): 1637-48.

29. Liu Yanhe, Chapple V, Feenan K, Roberts P, Matson P. Time-lapse deselection model for human day 3 in vitro fertilization embryos: the combination of qualitative and quantitative measures of embryo growth. Fertil Steril. 2016; 105 (3): 656-62.
3. Ombelet W, De Sutter P, Van der Elst J, Martens G. Multiple gestation and infertility treatment: registration, reflection and reaction-the Belgian project. Hum Reprod Update. 2005; 11: 3-14.

4. De Sutter P, Van der Elst J, Coetsier T, Dhont M. Single embryo transfer and multiple pregnancy rate reduction in IVF/ICSI: a 5-year appraisal. Reprod Biomed Online. 2003; 7: 464-9.

5. Thurin A, Hausken J, Hillensjo T, Jablonowska B, Pinborg A, Strandell A, Bergh C. Elective single-embryo transfer versus double-embryo transfer in in vitro fertilization. N Engl J Med. 2004; 
351: 2392-402.

6. Bergh C. Single embryo transfer: a mini-review. Hum Reprod. 2005; 20: 323-7.

7. Gardner DK, Meseguer M, Rubio C, Treff NR. Diagnosis of human preimplantation embryo viability. Hum Reprod Update. 2015; 21: 727-47.

8. Bavister BD. Culture of preimplantation embryos: facts and artifacts. Hum Reprod Update. 1995; 1: 91-148.

9. Meseguer M. Time-lapse: the remaining questions to be answered. Fertil Steril. 2016; 105 (2): 295-6.

10. Pribenszkya C, Matyasb S, Kovacsb P, Losonczic E, Zádori J, Vajta G. Pregnancy achieved by transfer of a single blastocyst selected by time-lapse monitoring. Reprod Biomed Online. 2010; 21 (4): 533-6.

11. Wang SX. The past, present, and future of embryo selection in in vitro fertilization: Frontiers in Reproduction Conference. Yale J Biol Med. 2011; 84 (4): 487-90.

12. McEvoy K, Brison D, Roberts S, Turner C, Adeniyi T, Wood L, et al. A one year retrospective analysis comparing live birth outcomes from embryos grown and transferred from an undisturbed timelapse culture system with a conventional culture system. Human reproduction. 2016; 31: i174-i175.

13. Shurygina OV, Saraeva NV, Tugushev MT, Pekarev VA, Bajzarova AA, Krasnova OV, et al. Otdalennye rezultaty programm VRT s ispolzovaniem sistemy nepreryvnogo videonabljudenija na jembriologichekom jetape. V sbornike: Reproduktivnye tehnologii segodnja i zavtra. Materialy XXVII mezhdunarodnoj konferenci Rossijskoj Associacii Reprodukcii Cheloveka; 6-9 centjabrja 2017 g. M.: Media Sfera, 2017; $328 \mathrm{~s}$.

14. Adamson GD, Abusief ME, Palao LM, Gvakharia M. Improved implantation rates of day 3 embryo transfers with the use of an automated time-lapse-enabled test to aid in embryo selection. Fertil Steril. 2015; 105: 369-75.

15. Diamond MP, Suraj V, Behnke EJ, Yang X, Angle MJ, LambeSteinmiller JC, et al. Using the Eeva Test ${ }^{\mathrm{TM}}$ adjunctively to traditional day 3 morphology is informative for consistent embryo assessment within a panel of embryologists with diverse experience. J Assist Reprod Genet. 2015; 32: 61-68.

16. Ver Milyea MD, Tan Lei, Joshua TA, Conaghan J, Ivani K, Gvakharia $\mathrm{M}$, et al. Computer-automated time-lapse analysis results correlate with embryo implantation and clinical pregnancy: A blinded, multi-centre study. Reprod Biomed Online. 2014; 29 (6): 729-36.

17. Kirkegaard K, Hindkjaer JJ, Grøndahl ML, Kesmodel US, Ingerslev HJ. A randomized clinical trial comparing embryo culture in a conventional incubator with a time-lapse incubator. J Assist Reprod Genet. 2012; 29 (6): 565-72.
18. Minasi MG, Colasante A, Riccio T, Ruberti A, Casciani V, Scarselli F, et al. Correlation between aneuploidy, standard morphology evaluation and morphokinetic development in 1730 biopsied blastocysts: a consecutive case series study. Hum Reprod. 2016; 31 (10): 2245-54.

19. Gardner DK, Schoolcraft WB. Culture and transfer of human blastocysts. Curr Opin Obstet Gynecol. 1999 Jun; 11 (3): 307-11.

20. Gardner DK, Lane M, Stevens J, Schlenker T, Schoolcraft WB. Blastocyst score affects implantation and pregnancy outcome: towards a single blastocyst transfer. Fertil Steril. 2000; 73: 1155-8.

21. Lee AM, Connell MT, Csokmay JM, Styer AK. Elective single embryo transfer - the power of one. Contracept Reprod Med. 2016; 1: 11

22. Goodman LR, Goldberg J, Falcone T, Austin C, Desai N. Does the addition of time-lapse morphokinetics in the selection of embryos for transfer improve pregnancy rates? A randomized controlled trial. Fertil Steril. 2016; 105 (2): 275-85.

23. Zhang JQ, Li XL, Peng Y, Guo X, Heng BC, Tong GQ. Reduction in exposure of human embryos outside the incubator enhances embryo quality and blastulation rate. Reprod Biomed Online. 2010; 20: 510-15.

24. Apter S, Ebner T, Freour T, Guns $\mathrm{Y}$, Kovacic B, Le Clef N, et al. Good practice recommendations for the use of time-lapse technology. Human Reproduction Open. 2020; 2020 (2: hoaa008. Available from: https://doi.org/10.1093/hropen/hoaa008.

25. Armstrong S, Bhide P, Jordan V, Pacey A, Marjoribanks J, Farquhar C. Time-lapse systems for embryo incubation and assessment in assisted reproduction. Cochrane Database of Systematic Reviews. 2018; 25 (5): CD011320. DOI: 10.1002/14651858. CD011320.pub4

26. Pribenszkya C, Nilselid AM, Montag M. Time-lapse culture with morphokinetic embryo selection improves pregnancy and live birth chances and reduces early pregnancy loss: a meta-analysis. Reprod Biomed Online. 2017 Nov; 35 (5): 511-520.

27. Meseguer M, Rubio I, Cruz M, Basile N, Marcos J, Requena A. Embryo incubation and selection in a time-lapse monitoring system improves pregnancy outcome compared with a standard incubator: a retrospective cohort study. Fertil Steril. 2012 Dec; 98 (6): 1481-9.

28. Athayde Wirka K, Chen AA, Conaghan J, Ivani K, Gvakharia M, Behr B, et al. Atypical embryo phenotypes identified by timelapse microscopy: high prevalence and association with embryo development. Fertil Steril. 2014; 101 (6): 1637-48.

29. Liu Yanhe, Chapple V, Feenan K, Roberts P, Matson P. Time-lapse deselection model for human day 3 in vitro fertilization embryos: the combination of qualitative and quantitative measures of embryo growth. Fertil Steril. 2016; 105 (3): 656-62. 